\title{
Playing dead during phage infection
}

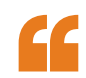

the trans-RNA
degradation
activity

of Cas 13

provides broad

immunity

against DNA

phages by

inducing a

dormant state

in infected cells

(1)

Bacteria and archaea have a variety of CRISPR-Cas systems that confer sequence-specific protection against invading plasmids and phages. Uniquely, the type VI effector nuclease Cas13 targets RNA instead of DNA, and catalyses both sequence-specific cis- and non-specific trans-RNA cleavage. Previous studies observed that type VI spacer sequences exclusively align with double-stranded DNA phages; however, the mechanism by which Cas13 uses its RNA cleavage activity to protect against DNA phages is not understood. Now, Meeske, Nakandakari-Higa and Marraffini show that trans-cleavage of both viral and host transcripts induces a state of dormancy in bacteria, which inhibits viral replication and the spread of CRISPR-resistant phages.

First, the authors investigated the ability of Cas 13 to provide defence against the DNA phage $\varphi R R 4$ in Listeria ivanovii by introducing a spacer library of $\varphi R R 4$ genes into an engineered strain that is susceptible

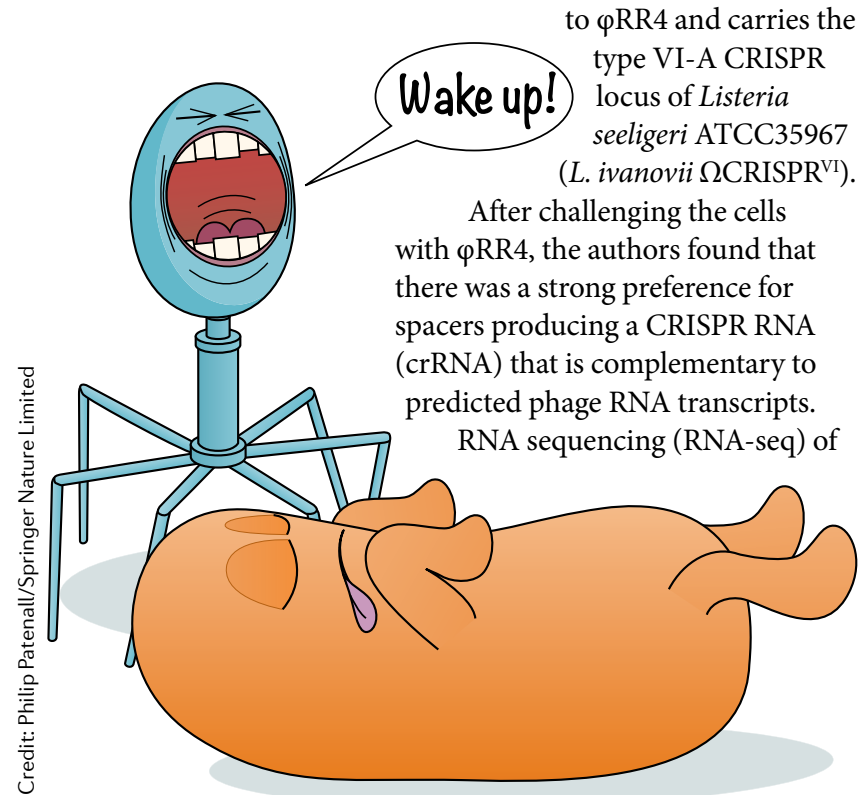

$\varphi$ RR4-infected cells found a marked correlation between protospacer transcription and protection conferred by the spacer. Indeed, the presence of one of three spacers significantly reduced $\varphi R R 4$ replication. Together, these results suggested that Cas 13 targets phage transcripts to block infection; however, the authors noticed that the function of the cis-targeted transcript appeared irrelevant and did not affect the potency of the response.

The authors hypothesized that Cas13-mediated trans-RNA degradation of both host and viral transcripts is important for type VI immunity. RNA-seq and global mapping of RNA 5' ends showed substantial degradation of host transcripts and early and late $\varphi R R 4$ transcripts during infection. Moreover, degradation of host transcripts was found when the expression of cas 13 was induced without infection.

Previous observations of bacterial growth defects during type VI immunity against plasmids suggested that host transcript degradation could shift infected cells into a state of dormancy, thus preventing phage propagation. In L. seeligeri ATCC35967, wild-type (but not $\triangle$ CRISPR) cells had an arrested growth phenotype after cas $13 a$ induction, which recovered once induction was stopped. These cells were viable, suggesting that Cas13a-induced dormancy is reversible. Furthermore, these dormant cells were resistant to transient exposures of bactericidal antibiotics that target growing cells. Moreover, cytological assays suggested that $L$. ivanovii $\Omega$ CRISPR ${ }^{\mathrm{VI}}$ cells entered into dormancy and remained viable after $\varphi R R 4$ infection. These results suggest that when Cas13 is activated by a target RNA, its RNase activity promotes a dormant state in the host, which is maintained in infected cells owing to the continued synthesis of protospacer RNA.

Next, the authors investigated whether the dormant state provides herd immunity to uninfected cells. Wild-type L. ivanovii RR3 ( $\varphi$ RR4sensitive) cells were protected from infection when co-cultured with $L$. ivanovii $\Omega$ CRISPR ${ }^{\mathrm{VI}}$ cells that encode a $\varphi R R 4$-specific spacer. The authors transformed a plasmid that carries a target into L. ivanovii $\Omega$ CRISPR ${ }^{\mathrm{VI}}$ cells (which carries a plasmid-targeting spacer, but not phage-targeting spacers). Pre-induction of cas $13 a$ using an inducible promoter resulted in an $\sim 6.7$-fold reduction in $\varphi \mathrm{RR} 4$ infection efficiency. In addition, the plasmid-targeting strain had a survival advantage after 7 hours of infection. The observation that a plasmid-activated type VI system protected against phage infection suggested that the system can provide broad, non-specific immunity. Subsequent experiments showed that phages harbouring escape mutations could be inhibited if the system was first activated by a wild-type phage, thus averting the rise of CRISPR-resistant phages.

Altogether, these observations support a model in which the trans-RNA degradation activity of Cas 13 provides broad immunity against DNA phages by inducing a dormant state in infected cells, thus thwarting phage replication and a viral epidemic.

Ashley York

ORIGINAL ARTICLE Meeske, A. J., NakandakariHiga, S. \& Marraffini, L. A. Cas13-induced cellular dormancy prevents the rise of CRISPR-resistant bacteriophage. Nature https://doi.org/10.1038/ s41586-019-1257-5 (2019) 